\title{
NOVE ESPÉCIES NOVAS DE ORTÓPTEROS DA FAMÍLIA PROSCOPIIDAE *
}

\author{
S. DE TOLEdo Piza JRL**
}

\begin{abstract}
Recebi dos professores A. Ferreira e A. Mesa, da Faculdade de Rio Clatro, para determinação, pequeno lote de Proscopiideos, do qual separei como novas para a ciência, as espécies que descrevo a seguir. Aos ilustres colegas agradeço a oferta dos tipos para o departamento do autor.
\end{abstract}

Tetanorfynchus proximus sp. $n$.

C - Caput versus oculos modice angustatum. Oculi elongati, prominentes. Fastigium parte reliqua capitis evidenter brevius, gracile, versus apicem angustatum, apice acuto, lateraliter et subter sulcatum, superne ad basim convexum. Antennae apicem fastigii fere attingentes, articulo proximali diametro oculorum breviore. Pronotum puncturatum, leviter corrugatum, utrinque luteum, superne obscurum. Mesonotum metanctumque subtiliter puncturato-corrugata. Abdomen partim subtilissime puncturatum. Lamina subgenitalis acuta, triplo longion quam altior, superne profunde excavata, inferne carinata lateraliter conveziuscula ac modicissime puncturata. Lamina supraanalis triangulariter elongata, extremitatem cercorum valde superans, superne puncturata ac sulcata. Femora gracilia, postica apice bispinosa dimidio proximali vix cratiora. Tibiae posticae marginibus superioribus multispinosae.

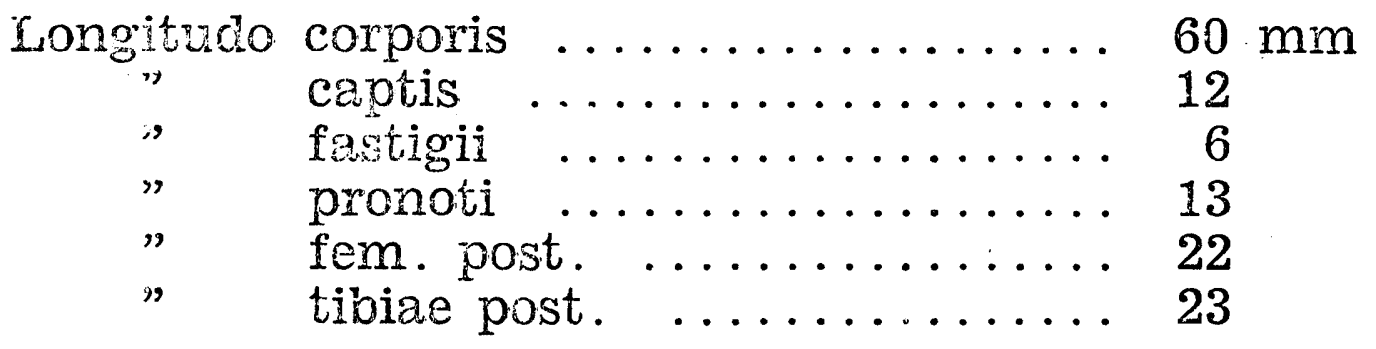

* Entregue para publicação em 25-3-77.

* Departamento de Zooiogia, ESALQ - USP. 
Pátria: Piracicaba (SP), Brasil

Col.: A. Mesa, XI - 1961

Tipo: Um macho, no Departamento de Zoologia da ESALQ, Piracicaba.

A presente espécie aproxima-se muito de Tetanorhynchus mendesi Piza, da qual se distingue pelas antenas quase atingindo o ápice do fastigio (bem mais distantes em mendesi) e pelo ápice da lâmina subgenital não bipartido.

Tetanorhynchus rostnatus sp. $n$.

$0^{x}$ - Caput elongatum, modice granulatum corrugatumque, oculis paulo prominentibus, fastigio gracili, acuto, parti capitis reliquae aequali, basi ruguloso, antennis trientem apicalem fastigii attingentibus, articulo I dimidio oculorum diametri minore. Pronotum capitis longitudine sat brevius, punctis ac rugulis obtectum, superne obscurum, lateraliter luteum. Mesonotum et metanotum pronoto similia. Abdomen puncturatum. Lamina subgenitalis punctata, basi humilis, apice acuto, bipartito, superne sulcata, inferne carinata. Femora gracilia. Tibiae posticae marginibus posterioribus serrulatis ac multispinosis, spinis apice nigris.

\begin{tabular}{|c|c|c|}
\hline Longitudo & corporis & $70 \mathrm{~mm}$ \\
\hline & capitis & 16 \\
\hline " & fastigii & 8 \\
\hline$"$ & pronoti & 14 \\
\hline$"$ & fem. post & 23 \\
\hline " & tib. post. & 24 \\
\hline
\end{tabular}

Pátria: Estado de São Paulo, Brasil.

Col.: A. Mesa, XI - 1961

T:ipo Um macho, no Departamento de Zoologia da ESALQ, Piracicaba.

Espécie muito semelhante a $T$. mendesi Piza, da qual se distingue pela cabeça evidentemente mais longa do que o pronoto.

Tetanorhynchus uruguaiensis sp. $n$.

$0^{x}$ - Caput postice quam antice vix cratius, indistincte granulatum, oculis paulo prominentibus. Fastigium forte, versus apicem modice angustatum, apice rotundatum, parte reliqua capitis sat brevius, dia- 
metro longitudinali oculorum minus quam duplo longius, superne convexiusculum, lateraliter et subter modice depressum. Duae partes distales segmenti ultimi antennarum apicem fastigii superantes, segmento I dimidio oculorum diametro subaequali, pronotum capite paulo longius teres, haud carinatum, levissime corrugatum, modicissime puncturatum. Lamina supraanalis angusta, apice acuto extremitem cercorum paulo superanti, superne longa. Lamina subgenitalis acuta, fastigio subaequali superne canaliculata, inferne carinata, utrinque subplana. Pedes breves, graciles. Tibiae III multispinosae. Coloreluteo-viridi, pronoto femoribusque ferrugineo maculatis .

\begin{tabular}{|c|c|c|}
\hline Longitudo & corporis & $54 \mathrm{~mm}$ \\
\hline & capitis & 10 \\
\hline$"$ & fastigii & 3,2 \\
\hline$"$ & pronoti & 11 \\
\hline$"$ & fem. post & 18,5 \\
\hline$"$ & tib. post. & 19,0 \\
\hline
\end{tabular}

Pátria: San Gregorio Artigas, Uruguai

Col.: A. Mesa e P. San Martin, 23-III-1959

Tipo: Um macho, no Departamento de Zoologia da ESALQ, Piracicaba.

Parátipo: Um macho, mesma procedência e data e os mesmos colecionadores.

Difere das espécies afins pelo comprimento bem menor dos femures e tibias posteriores.

Tetanorhynchus montanus sp. $n$.

$\sigma^{\pi}$ - Caput post oculos teres, ad basim modice crassius, parce puncturatum, oculis clongatis, pualo prominentibus, fastigio angusto, apicem versus modice graciliore, modicissime arcuato, superne omnino fere convexo, obsolete puncturato, apice rotundato, lateraliter et subter subplano, marginibus vix prominentibus, ciametro oculorum duplo fere longiore, parte reliqua capitis evidenter breviore. Ant,ennae apicem fastigii fere attingentes, articulo primo dimidio oculorum diametro breviore. Pronotum capite longius, dense puncturatum, margine antico rotundato, granulis nigris duobus simetricis praedito. Segmenta abdominalia puncturato-granulata. Lamina supraanalis angusta, apice acuto, extremitatem cercorum superante, puncturata, indistincte foveolata. Lamina subgenitalis longitudini fastigii aequilonga, humilis, acuta, crasse puncturata, facieis planis, marginibus angulosis, inferne carinata, supernf n tantum carinulata. Femora postica cylin- 
dracea, versus basim indistincte crassiora. Tibiae superne multispinosae. latum.

Corpus olivaceum, parte ventalis dilutiore, dense obscure macu-

Longitudo corporis ............ $64 \mathrm{~mm}$

$\begin{array}{lllr}" & \text { capitis } \ldots \ldots \ldots \ldots \ldots \ldots \ldots \ldots & 12 \\ " & \text { fastigii } \ldots \ldots \ldots \ldots \ldots \ldots \ldots \ldots & 5 \\ " & \text { pronoti } \ldots \ldots \ldots \ldots \ldots \ldots \ldots & 14 \\ " & \text { fer. post. } \ldots \ldots \ldots \ldots \ldots \ldots & 21 \\ " & \text { tib. post. } \ldots \ldots \ldots \ldots \ldots \ldots & 23\end{array}$

Pátria: Serra da Mantiqueira (M.G.), Brasil.

Col.: A. Mesa, 31-XII-61

Tipo: Um macho, no Departamento de Zoologia da ESALQ, Piracicaba.

Parátipos: Dois machos, mesma procedência e mesmo colecionador.

Aproxima-se de $T$. gracilis Brunner, do qual se distingue pelas antenas quase atingindo o ápice do iastigio, pelo pronoto mais longo do que a cabeça, lâmina subgenital igual ao fastigio e pelas manchas escuras do corpo.

Teianorhynchus bolivicnus sp.n.

$\sigma^{\pi}$ - Robustus. Ceput regulariter conicum, crassum, subtiliter puncturatum, oculis paulo prominentibus, antennis apicem fastigii at tingentibus. Fastigium forte, acutum, modicissime arcuatum, superne convexum, marginibus haud prominentibus, planum, marginibus inferioribus modice prominentibus, oculis duplo longius, parte reliqua capitis evidenter brevius. Pronotum subtiliter dense puncturatum, capice paulo brevius. Lamina supraanal lata, apice obtuse rotundata, indisdincte foveolata. Lamina subgenitalis minus quam duplo longior quam altior, superne late canaliculata, inferne carinata, fastigio brevior. Femora postica robusta, spinulis apicalibus parvis. Tibiae superne multispinosae.

Corpus olivaceum, capite longitudinaliter obscuriter vittato, pronoto in medio vitta lata ocracea. 
Longitudo corporis $\ldots \ldots \ldots \ldots \ldots \ldots, 69 \mathrm{~mm}$

$\begin{array}{lllr}" & \text { capitis } \ldots \ldots \ldots \ldots \ldots \ldots \ldots \ldots & 13 \\ " & \text { fastigii } \ldots \ldots \ldots \ldots \ldots \ldots \ldots & 6 \\ " & \text { pronoti } \ldots \ldots \ldots \ldots \ldots \ldots \ldots & 15 \\ " & \text { fem. post. } \ldots \ldots \ldots \ldots \ldots \ldots & 23 \\ " & \text { tib. post. } \ldots \ldots \ldots \ldots \ldots \ldots & 25\end{array}$

Pátria: Santa Cruz de La Sierra, Bolivia.

Col.: A. Mesa, IV-1962.

Tipo: Um macho, no Departamento de Zoologia da ESALQ, Piracicaba

Parátipo: Um macho da mesma procedência e data.

Aproxima-se de $T$. bihastatus Rehn, diferindo pelos olhos menos proeminentes, pelo fastígio mais longo, iguall a duas vezes o diâmetro longitudinal dos olhos e pelos fêmures posteriores mais espêssos. Aproxima-se também de $T$. Rileyi, do qual se aistíngue pelo colorido, e pela forma do fastígio e da lâmina subgenital.

Tetanorhynchus carbonelli sp. $n$.

$0^{x}$ - Gracilis. Caput cylindro-conicum, basi paulo ampliatum, oculis elongatis, non multo prominentibus, fastigio apicem versus vix angustiore, apice obtuso, minus quam duplo oculorum diametro longiore, superne puncturato, marginibus rotundatis paulo prominentibus, inferne canaliculato, dimidio partis reliquae capitis vix longiore. Antennae apicem fastigii vix superantes, aticulo basilari quarta parte diametri oculorum haud majore. Pronotum fortiter puncturatum, capite sesqui longius, in medio indistincte vel modice carinulatum. Abdomen in medio carinulatum. Pars terminalis abdominis brevis. Femora postica gracilia, tibiae multispinosae.

Corpus et femora postica obscure maculata.

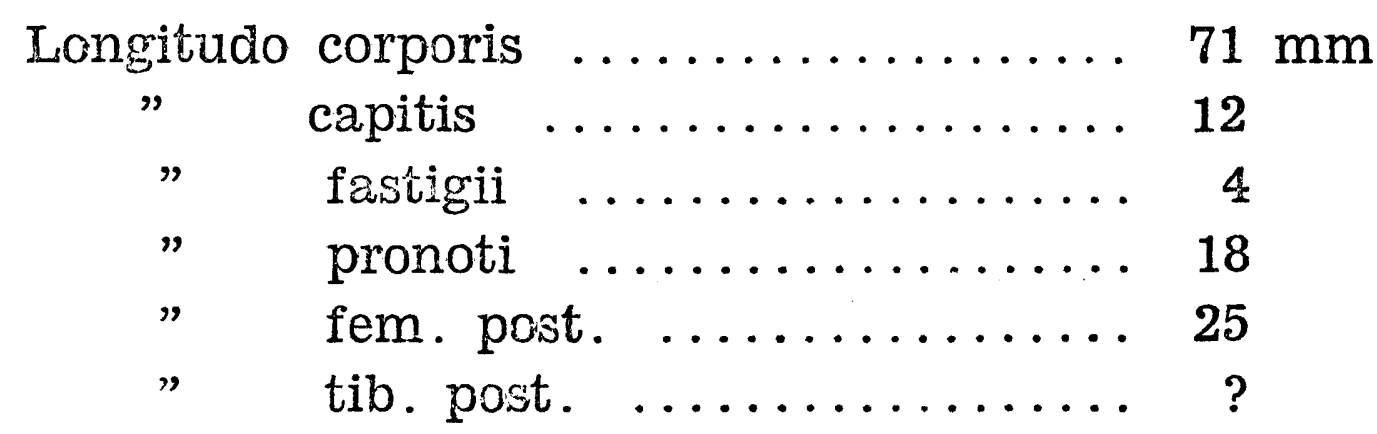


Pátria: Rio Grande do Sul (Nonoai), Brasil.

Col.: C. Carbonell, A. Mesa e M.A. Monné, 20-II-1964.

Tipo: Um macho no Departamento de Zoologia da ESALQ, Piracicaba.

Parátipo: Um macho da mesma procedência, colecionado na mesma data.

Difere de T. humilis Giglio-Tos pelo aspecto geral e pela coloração.

Cephalocoema caaguazu sp. $n$.

- Robusta. Caput a basi usque ad fastigii apicem regulariter conicum. Oculi sat humiles, elongati. Articulus I antennarum dimidium oculorum diametro vix superans. Fastigium forte, conicum, apice obtusum, superne convexum, parce minute puncturatum, marginibus rotundatis haud prominentibus, lateraliter in medio obtusissime carinatum, inferne late sulcatum, oculorum diametro circa duplo longius. Pronotum cylindraceum, capite haud longius, subtiliter puncturato-granulatum. Abdomen levissime puncturatum. Lamina supraanalis triangularis, acuta, foveolata ac puncturata extremitatem cercorum valde superante. Valvae ovipositoris parallelae, plus quam duplo longiores quam latiores, parce puncturatae, marginibus superioribus obsolete denticulatis. Femora postica versus basim paulo crassiora, spinulis apicalibus minutissimis. Tibiae multispinosae.

Caput lateraliter late luteo vittatum. Mesonotum metanotum que utrinque nigro-vittata .

$\sigma^{\pi}$ - Feminae similis sed minor, antennis fastigio longioribus, lamina subgenitali fastigio longiore, superne lata, marginibus obsoletissime nodulosis, inferne obtuse carinata, apice acuto.

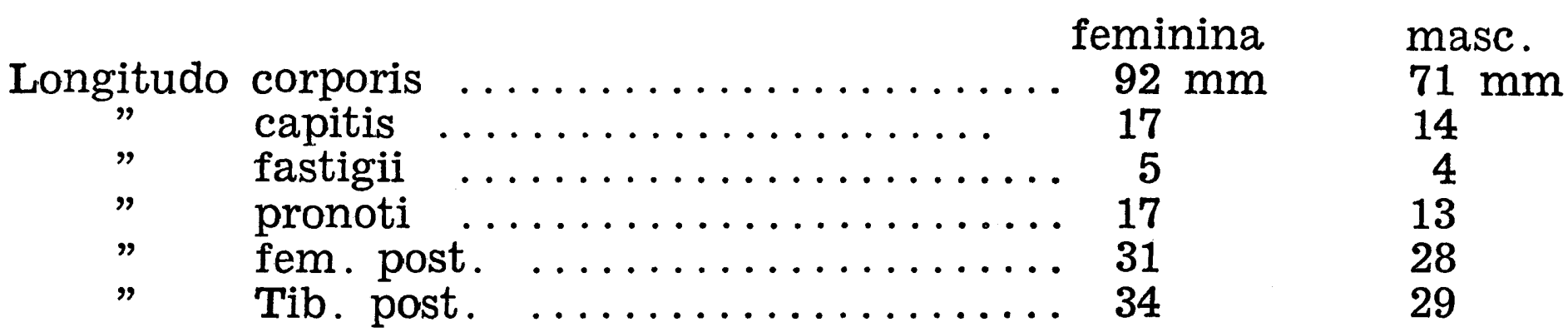

Pátria: Caaguazú, Paraguai

Col.: C.S. Carbonell, A. Mesa e M.A. Monné, 13-III-65

Tipo: Uma femea, no Departamento de Zoologia da ESALQ, Piracicaba. Alótipo: Um macho de mesma procedência, col. na mesma data, no mesmo departamento. 
Assemelha-se à $C$. multispinosa Brunner diferindo pela coloração, por ser maior, a fêmea com o fastigio de bordos lisos, faces laterais não escavadas, pronoto, mesonoto e metanoto destituidos de crista mediana, menor do que a metade do resto da cabeça; macho difere pelo comprimento da lâmina subgenital, que é maior que o fastígio.

Cephalocoema gaucha sp. $n$.

$\sigma^{\top}$ - Gracilis. Caput elongatum, pronoto evidenter longius, obsoletissime carinatum, carinis granulatis, oculis elongatis, sat humilibus, fastigio longo, capite duplo breviore, superne omnino fere convexo, marginibus obtusis, extremitatem versus sensim graciliore, utrinque obtuse carinato, inferne deplanato, marginibus rotundatis, antennis fastigio paulo brevioribus, articulo basilari dimidio oculorum longiore. Pronotum breve, indistincte carinatum, leviter granulato-puncturatum. Mesonotum, metanotum ac segmentum medianum tricarinata, carinis lateralibus castaneo-nigris. Abdomen carinulatum. Lamina supraanalis triangularis, acuta, sulcata. Lamina subgenitalis fastigio evidenter longior, gracilis, acuta, marginibus superioribus rotundatis, indistincte nodulosis, inferne obtuse carinata, superne plus minusve deplanata, carinataque. Pedes graciles. Femora postica spinis apicalibus nullis. Pronotum utrinque castaneo-ferrugineo vittatum, ad suturas luteum, infra suturas casteneo-ferrugineum, subter lutescenti. Pars ventralis corporis diluta.

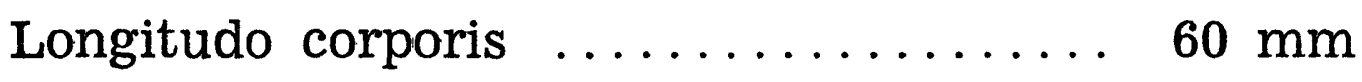

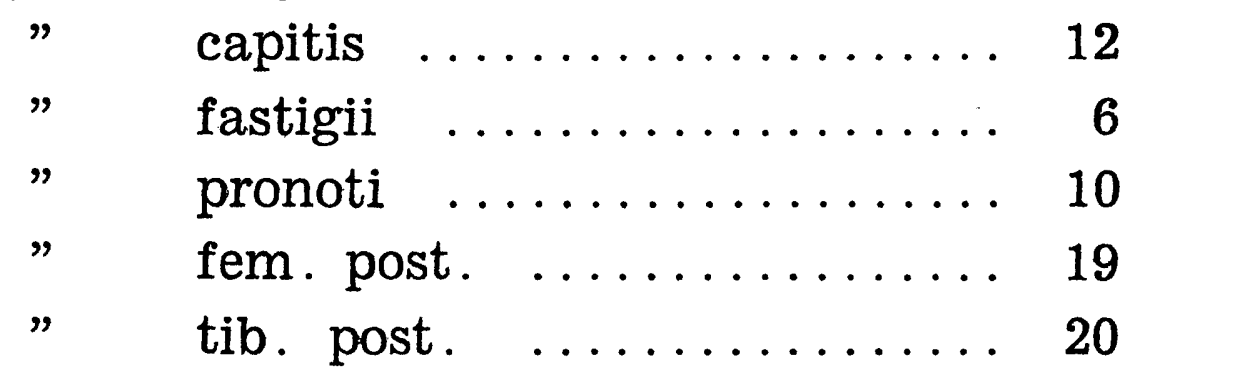

Pátria: Rio Grande do Sul

Col.: C.S. Carbonell, A. Mesa e M.A. Monné, II - 1964.

Tipo: Um macho, no Departamento de Zoologia da ESALQ, Piracicaba.

Parátipos: Quatro machos da mesma procedência.

Aproxima-se de $C$. chapadensis Rehn, da qual se distingue pelo fastigio igual ao resto da cabeça, as antenas mais curtas que o fastigio, pronoto menor que a cabeça, lâmina supraanal larga e de ápice agudo e pela coloração. 
Cephalocoema meridialis $s p . n$.

$0^{*}$ - Gracilis. Caput post oculos modice angustatum, rusticum. Oculi longi, humiles. Fastigium forte, apicem versus modice angustatum, apice rotundato, superne convexum, caelatum, marginibus paulo productis, lateraliter modice canaliculatum et in medio obtuse carinatum, inferne planum, dimidio partis reliquae capitis brevius. Antennae fastigio fere duplo longiores, articulo proximali dimidio oculorum diametri longiore. Pronotum capite brevius, fortiter puncturatum, marginibus puncturatum partim confluentibus, obsoleter carinatum. Abdomen indistincte puncturatum, carinulatum. Lamina supraanalis triangularis, elongata, apice obtuso, sulcata. Lamina subgenitalis fastigio duplo longior, superne concava, lateraliter subplana, inferne modice carinata. Femora postica gracilia, apice haud spinulosa. Tibiae multis pinosae.

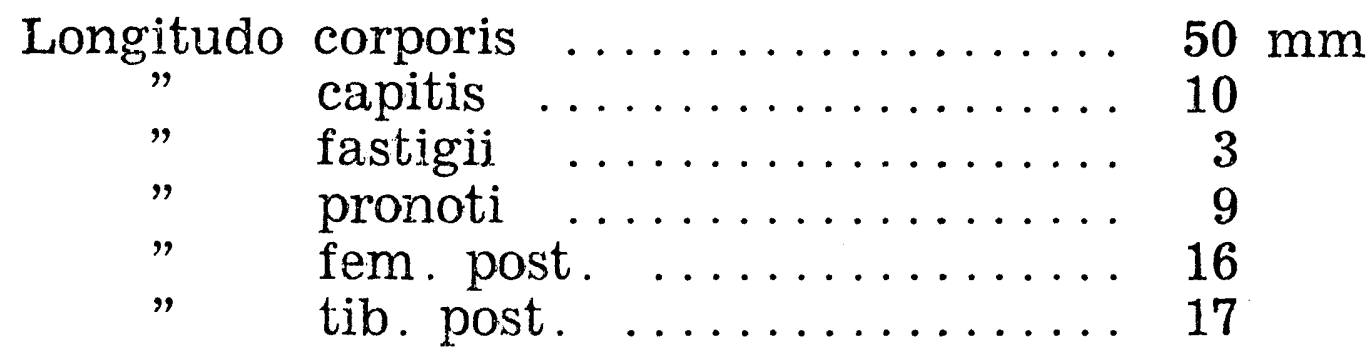

Pátria: Rio Grande de Sul.

Col.: C. Carbonell, A. Mesa e M.A. Monné, II-1964.

Tipo: Um macho no Departamento de Zoologia da ESALQ, Piracicaba.

Difere de $C$. multispinosa Brunner pelo fastigio muito menor que o resto da cabeça, pelo pronoto mais curto que a cabeça e pela lâmina subgenital bem mais longa do que o fastigio. 